\title{
Characterization of Tetraploid Somatic Cell Nuclear Transfer-Derived Human Embryonic Stem Cells
}

\author{
Dong-Hyuk Shin ${ }^{1,2}$, Jeoung-Eun Lee ${ }^{3}$, Jin Hee Eum ${ }^{1}$, Young Gie Chung ${ }^{3}$, \\ ${ }^{\dagger}$ Hoon Taek Lee ${ }^{2}$ and ${ }^{\dagger}$ Dong Ryul Lee ${ }^{3,4}$ \\ ${ }^{1}$ Fertility Center of CHA Gangnam Medical Center, College of Medicine, CHA University, Seoul 06135, Korea \\ ${ }^{2}$ Dept. of Animal Biotechnology, Konkuk University, Seoul 05020, Korea \\ ${ }^{3}$ CHA Stem Cell Institute, CHA University, Seongnam 13488, Korea \\ ${ }^{4}$ Dept. of Biomedical Science, College of Life Science, CHA University, Seongnam 13488, Korea
}

\begin{abstract}
Polyploidy is occurred by the process of endomitosis or cell fusion and usually represent terminally differentiated stage. Their effects on the developmental process were mainly investigated in the amphibian and fishes, and only observed in some rodents as mammalian model. Recently, we have established tetraploidy somatic cell nuclear transfer-derived human embryonic stem cells (SCNT-hESCs) and examined whether it could be available as a research model for the polyploidy cells existed in the human tissues. Two tetraploid hESC lines were artificially acquired by reintroduction of remained 1st polar body during the establishment of SCNT-hESC using MII oocytes obtained from female donors and dermal fibroblasts (DFB) from a 35-year-old adult male. These tetraploid SCNT-hESC lines (CHA-NT1 and CHA-NT3) were identified by the cytogenetic genotyping (91, XXXY,-6, t[2:6] / 92,XXXY,-12,+20) and have shown of indefinite proliferation, but slow speed when compared to euploid SCNT-hESCs. Using the eight Short Tendem Repeat (STR) markers, it was confirmed that both CHA-NT1 and CHA-NT3 lines contain both nuclear and oocyte donor genotypes. These hESCs expressed pluripotency markers and their embryoid bodies (EB) also expressed markers of the three embryonic germ layers and formed teratoma after transplantation into immune deficient mice. This study showed that tetraploidy does not affect the activities of proliferation and differentiation in SCNT-hESC. Therefore, tetraploid hESC lines established after SCNT procedure could be differentiated into various types of cells and could be an useful model for the study of the polyploidy cells in the tissues.
\end{abstract}

Key words : Polyploidy, Tetraploidy, Somatic cell nuclear transfer, Human embryonic stem cells

\section{INTRODUCTION}

Polyploidy, such as triploidy or tetraploidy, is a condition with increased whole chromosome sets in cells, and it is an important topic in cell biology. This process typically occurs during endomitosis or cell fusion and results in terminally differentiated cells, such as megakaryocytes or embryonic trophoblasts (Edgar \& Orr-Weaver, 2001). In particular, tetraploidy is thought to arise from multipolar mitosis, progressive chromosomal loss, and chromosome mis-segregation during mitosis. Subsequently, tetraploid cells give rise to aneuploid daughter cells, because of

\footnotetext{
Manuscript received November 1, 2017, Received in revised form November 22, 2017, Accepted November 30, 2017

${ }^{\dagger}$ Corresponding Author : Dong Ryul Lee, Ph.D., Department of Biomedical Science, College of Life Science, CHA University, 335 Pankyo-ro, Seongnam 13488, Korea. Tel: +82-31-881-7136, Fax: +82-31-881-7100, E-mail: drleedr@cha.ac.kr

Hoon Taek Lee, Ph.D., Department of Animal Biotechnology, Konkuk University, 120 Neungdong-ro, Gwangjin-gu, Seoul 06135, Korea. Tel: +82-2457-8488, Fax: +82-2-457-8488, E-mail: ht13675@konkuk.ac.kr

This is an Open Access article distributed under the terms of the Creative Commons Attribution Non-Commercial License (http:// creativecommons.org/licenses/by-nc/3.0) which permits unrestricted non-commercial use, distribution, and reproduction in any medium, provided the original work is properly cited.
} 
cytokinesis failure (Ganem et al., 2007; Lv et al., 2012). Also, tetraploidy has a high probability of possessing more than double the amount of spontaneous DNA damage and hence, an increased requirement for DNA repair processes (Mable \& Otto, 2001; Storchova \& Kuffer, 2008). Even if the amount of DNA damage does not increase significantly, the processes for repair might be less efficient in tetraploidy. Some studies have described that both yeast and mammalian tetraploid cells appear to be more sensitive to DNA-damaging agents than euploid cells (Hau et al., 2006; Storchova et al., 2006).

It has been recently suggested that tetraploidy, like polyploidy, is detrimental to early mammalian development (Imai et al., 2015; Kawaguchi et al., 2009). The cell cycle in a tetraploid mouse embryo is prolonged, and it induces a reduction in the number of cells and results in malformation and abnormal cessation of embryonic development (Koizumi \& Fukuta, 1995). Actually, polyploid cells are frequently found in tumors of all stages, and several reports support the idea that tetraploid cells occur as an early step in tumor formation (Storchova \& Kuffer, 2008) and cancer (Mazumdar et al., 2006; Duelli et al., 2007). For this reason, it has been suggested that polyploidy is formed from spontaneous miscarriages and could be used as a biomarker for the diagnosis of cancer (Mandrioli et al., 2016). In addition, a tetraploid human is thought to be a rare clinical case that results in high intrauterine lethality in humans, because of a genetic aberration (it is found in $1-2 \%$ of early miscarriages); only a few clinical reports of infants diagnosed with tetraploidy are available. In fact, the most common outcome of the tetraploid human fetus is miscarriage, and even if the pregnancy reaches full term, the infant dies shortly after birth (Bothur-Nowacka et al., 2013). Most delivered babies have died between birth and one year of age. The oldest recorded non-mosaics tetraploid patient was 26-months-old (Guc-Scekic et al., 2002), while another female was at least 22-months-old (Lafer \& Neu, 1988). Interestingly, in humans, mosaic polyploidy in some parts of specific tissues occurs more frequently than polyploidy in the whole body. Generally, mosaic polyploidy may affect cell development. Also, it has been recently suggested that established tetraploidization in oncogenes is linked to tumorigenic potential (Storchova \& Kuffer, 2008). In addition, the spontaneous tetraploidization of primary cells from patients diagnosed with Gardner syndrome was observed several decades ago (Danes, 1976). On the other hand, some reports have suggested that the relatively frequent presence of polyploid cells in normal tissues ( $0.5-20 \%$ depending on the tissue) brings some advantages for the function of tissue differentiation or for functional adaptation (Biesterfeld et al., 1994). However, there is a limitation in the study of polyploidy in the human system, because polyploid human and mammals are very rare cases (Imai et al., 2015), although it is very common among plants, fish, and amphibians in nature. So, in the present study, we have examined the characteristics and function of tetraploid SCNT-hESCs, and believe that this could be developed as a research model for tetraploid cells that exist in human tissues.

\section{MATERIALS AND METHODS}

\section{Preparation of human oocytes}

The study protocol (CHA001) was approved by both the CHA Regenerative Medicine Institute (CHARMI) Stem Cell Research Oversight (SCRO) Committee and the Essex Institutional Review Board (EIRB). Ovarian stimulation protocol of anonymous egg donors was followed previous report (Chung et al., 2014). The collected cumulus-oocytes complexes (COCs) were denuded with 50-80 IU/mL hyaluronidase (Sigma-Aldrich) within 1-2 hours after retrieval. Then, they were kept either in Cleavage medium supplemented with $10 \%$ serum substitute supplement (SSS; Cooper Surgical) or Global medium 10\% (IVF Online) until use. 


\section{Nuclear donor cell preparation and characterization}

Human dermal fibroblast (DFB) cells were obtained from normal healthy male volunteers (46 XY). Donor was 35 years old (DFB-1). Small pieces of skin from the inner forearm $(0.5 \mathrm{~cm} \times 0.3 \mathrm{~cm})$ were biopsied under local anesthesia and washed 3 times in PBS supplemented with an antibiotic/antimycotic solution (anti-anti 1×, Invitrogen) to remove any possible contaminants. Nuclear donor cell preparation and characterization was followed previous report (Chung et al., 2014).

\section{Somatic cell nuclear transfer procedure and em-} bryo culture and derivation of hESCs from cloned blastocysts

Human tetraploid SCNT-hESCs can be acquired during traditional SCNT procedure under an inverted microscope equipped with a Polscope (Oosight, Cambridge Research \& Instrumentation, UK) to visualize the spindle complex (Fig. 1). All MII oocytes were pre-incubated in Quinn's Advantage medium with HEPES (Cooper Surgical) containing caffeine $(1.25 \mathrm{mM})$ and $0.5 \mu \mathrm{g} / \mathrm{mL}$ cytochalasin $\mathrm{B}$ for 5 minutes. For the enucleation, a holding pipette was used to rotate each oocyte so that the spindle complex was positioned between 5 and 6 o'clock. The zona pellucida below the spindle was cut by a "knife pipette" and a small volume of cytoplasm containing MII spindle was extruded by applying a gentle squeezing force. After the removal of spindle complex, an isolated DFB cell was aspirated into an insertion pipette, transferred, resuspended in a drop containing HVJ-E extract (Isihara Sangyo Kaisha, Japan), and inserted into the perivitelline space of the enucleated oocytes. Differ from traditional SCNT procedure, tetraploid SCNT-hESCs were generated by refusion of remained 1st polar body during the procedure (Fig. 1B).

Once cell fusion was confirmed, the reconstructed eggs were transferred into Quinn's cleavage medium 10\% SPS, and incubated for 2 hours before activation in an incubator with atmosphere of $5 \% \mathrm{CO}_{2} / 5 \% \mathrm{O}_{2} / 90 \% \mathrm{~N}_{2}$ at $37^{\circ} \mathrm{C}$.
Activation of the reconstructed eggs was carried out using ionomycin $(5 \mu \mathrm{M}, 5 \mathrm{~min})$ and 6-dimethyl aminopurine (6DMAP, $2 \mathrm{mM}, 4 \mathrm{hrs}$ ) in Cleavage medium 10\% SPS (Cooper Surgical). The cloned embryos were cultured in Global 10\% FBS medium containing TSA (10 nM, Sigma) for $12 \mathrm{hrs}$ after activation, whereupon embryos were transferred to Global 10\% FBS without TSA and culture. The culture medium was switched to G2 medium supplemented with $10 \%$ HSA (Vitrolife) at day 2 and embryos were cultured up to blastocyst by day 6 .

After removal of the zona pellucida with acidic Tyrode's solution, whole blastocysts (without removing trophectoderm) were plated onto mitotically-inactivated mouse fibroblasts (MEFs, Global Stem Inc. Rockville, MD) in knockout DMEM-based human ESC derivation medium composed of knockout-DMEM supplemented with Knockout Serum Replacement (10\% SR, Invitrogen), FBS (10\% Hyclone), bFGF (30 ng/mL), and human LIF (2,000 units/ $\mathrm{mL}$, Sigma-Aldrich) (Chung et al., 2008).

\section{Chromosome analysis and nuclear DNA genotyping}

Karyotyping for both NT-cell lines were performed using a standard protocol. Metaphase spreads were stained with a GTG (G-bands by trypsin using Giemsa)-banding technique and 20 metaphases were analyzed and karyotyped by two cytogenetics experts. The ideogram was produced by the Ikaros karyotyping system (MetaSystems, Germany).

Nuclear DNA genotyping of NT-ESCs, donor fibroblasts, and donor oocytes was performed by using AmpFlSTR $^{\circledR}$ identifier kit (Applied Biosystems). PCR reactions were performed according to the manufacturer's protocol. The PCR products were loaded on ABI 3130 genetic analyzer (Applied Biosystems) and analyzed by the GeneMapper ${ }^{\circledR}$ ID Software v3.2.1 (Applied Biosystems).

\section{Immunocytochemistry of tetraploid SCNT-hESCs} and its EB

To confirm hESC-specific marker expression, tetraploid 
SCNT-hESCs were cultured with mitomycin-C-treated MEFs on a cover slip. After $48 \mathrm{hrs}$ of culture, the cells were fixed with $4 \%$ paraformaldehyde at $4{ }^{\circ} \mathrm{C}$ for overnight washed 1 $\times$ phosphate buffered saline (PBS, Hyclone) each $15 \mathrm{~min}$ washing. After permeabilization by $0.1 \%$ Triton-X 100 in PBS for 10 min and blocking with protein block RT for 60 min, the cells were incubated $4{ }^{\circ} \mathrm{C}$ for overnight with primary antibodies against OCT-3/4 (SantaCruz sc-5279, 1: 100), SSEA-4, TRA 1-60 and TRA-1-81 (Millipore MAB 4304, MAB4360, MAB4381, 1:100), and Alexa Flour 555 goat anti-mouse IgG antibody (Molecular probes A21424, 1:200) for $1 \mathrm{hr}$ at room temperature and All samples were counterstained with $1 \mu \mathrm{g} / \mathrm{mL}$ DAPI (Sigma-Aldrich) for 15 min at room temperature and mounted using vector shield mounting medium (Vector laboratories, Inc,. Burlingame, CA). Sample images were captured with a Axio Imager 2 (Carl Zeiss, Jena, Germany).

The differentiation capacity of tetraploid SCNT-hESCs line was confirmed by EB formation in vitro For EB formation, tetraploid SCNT-hESCs were culture in suspension without human bFGF for 2 weeks, and fixed with $4 \%$ paraformaldehyde at $4{ }^{\circ} \mathrm{C}$ for overnight after $1.5 \%$ Agarose embedding and re-fixed with $4 \%$ paraformaldehyde at $4{ }^{\circ} \mathrm{C}$ for overnight After washing, the tissues were dehydrated with a $70 \%, 80 \%, 90 \%, 95 \%$ and twice $100 \%$ alcohol series and twice $100 \%$ Xylenec (Clearing), then embedded in paraffin blocks. The paraffin-embedded blocks were serially sectioned at $4 \mathrm{~mm}$. Four-micrometer sections of the paraffin-embedded tissue arrays were deparaffinized, rehydrated in a graded series of alcohol and microwavetreated for $30 \mathrm{~min}$ in a target retrieval solution (Dako North AmeriInc., Carpinteria, CA). This process was repeated three times. Sections were blocked with protein blocking solution (Dako) for $1 \mathrm{hr}$ at room temperature. Then, slides were stained overnight at $4{ }^{\circ} \mathrm{C}$ with primary antibodies, then the differentiation of EBs into three-germ layers was confirmed by immunohistochemistry. Antibo- dies against AFP (Alpha-1-Fetoprotein; Dako A0008, 1: 100) for endoderm, 'aSMA (alpha smooth muscle actin; abcam ab5694, 1:100), for medoderm, and Tuj1 (B-Tubulin; Covance PRB-435P, rabbit, 1:100), for ectoderm were used. A Alexa Flour 555 goat anti-rabbit IgG antibody (Molecular probes A21420) was used as a secondary antibody diluted to 1:100 with DPBS (Hyclone) for $1 \mathrm{hr}$ at room temperature in dark. All samples were counterstained with $1 \mu \mathrm{g} / \mathrm{mL}$ DAPI (Sigma-Aldrich) diluted 1: 500 with DPBS (Hyclone) for $15 \mathrm{~min}$ at room temperature and mounted using vector shield mounting medium (Vector laboratories, Inc,. Burlingame, CA). Sample images were captured with a Axio Imager 2 (Carl Zeiss, Jena, Germany).

\section{Teratoma formation}

The differentiation capacity of tetraploid SCNT-hESCs line was confirmed by, approximately $1 \times 10^{5}$ of undifferentiated tetraploid SCNT-hESCs were injected into the testicle of a NOD/SCID male mouse. For each tetraploid SCNT-hES cell line, at least 3 animals were used. After 14 16 weeks, teratomas were excised, fixed in $4 \%$ paraformaldehyde at $4{ }^{\circ} \mathrm{C}$ for 2 week, trimming after re-fixed with $4 \%$ paraformaldehyde at $4{ }^{\circ} \mathrm{C}$ for 1 week after washing, the tissues were dehydrated with a $70 \%, 80 \%, 90 \%$, $95 \%$ and twice $100 \%$ alcohol series $1 \mathrm{hr}$ at room temperature and twice $100 \%$ Xylene, then embedded in paraffin blocks. The paraffin-embedded blocks were serially sectioned at $4 \mu \mathrm{m}$. Four-micrometer sections of the paraffin-embedded tissue arrays were deparaffinized, rehydrated in a graded series of alcohol and microwave-treated for $30 \mathrm{~min}$ in a target retrieval solution (Dako North AmeriInc., Carpinteria, CA). This process was repeated three times. Sections were blocked with protein blocking solution (Dako.) for $1 \mathrm{hr}$ at room temperature. Then, slides were stained overnight at $4{ }^{\circ} \mathrm{C}$ with primary antibodies, then the differentiation of teratoma formation into three germ layers was confirmed by immunohistochemistry. 
Antibodies against Tuj1 (B-Tubulin; Covance PRB-435P, rabbit, 1:100), for ectoderm were used. A Alexa Flour 555 goat anti-rabbit IgG antibody (Molecular probes A21420) was used as a secondary antibody diluted to 1:100 with DPBS for $1 \mathrm{hr}$ at room temperature in dark. All samples were counterstained with $1 \mu \mathrm{g} / \mathrm{mL}$ DAPI (Sigma-Aldrich) diluted 1: 500 with DPBS for $15 \mathrm{~min}$ at room temperature and mounted using vector shield mounting medium. Alcian Blue stain for Cartilage and Masson`s trichrome stain for medoderm and periodic acid-schiff (PAS) stain for Secretory epithelium (endoderm) staining Sample images were captured with a Axio Imager 2 (Carl Zeiss, Jena, Germany).

\section{Reverse transcription-polymerase chain reaction}

To confirm hESC-specific marker expression, RT-PCR was performed using primer sets (SOX2, F: 5' - agc tac agc atg atg cag ga - 3', R: 5' - ggt cat gga gtt gta ctg ca - 3', 125 bp; OCT3/4, F: 5' - gca att tgc caa gct cct gaa gca g 3', R: 5' - cat agc ctg ggg tac caa aat ggg g - 3', 536 bp; NANOG, F: 5' - tga acc tca gct aca aac ag - 3', R: 5' - tgg tgg tag gaa gag taa ag - 3', $153 \mathrm{bp} ; G A P D H, \mathrm{~F}: 5^{\prime}$ - aga agg ctg ggg ctc att tg - 3', R: 5' - agg ggc cat cca cag tct tc - 3', $258 \mathrm{bp}$ ). And to confirm the three germ layers differentiation in $\mathrm{hEB}$, used primer sets for ectoderm ( $S O X 1$, F: 5' - gtg tcc aat tgt tgg cat cta ggt c - 3', R: 5' - caa gga aat aag gtg gtt gga gca c - 3', 202 bp; NEUROD1, F: 5' gtt ctc agg acg agg agc ac - 3', R: 5' - tgg aca gct tct gcg tct ta - 3', 333 bp), mesoderm (ACTC1, F: 5' - cat cct gac cct gaa gta tcc cat c - 3', R: 5' - ccc tca tag atg ggg aca ttg tga g - 3', 315 bp; HAND1, F: 5' - aag aga acc aga cgc agg aa - 3', R: 5' - ggc agg atg aac aaa cac ct - 3', 263 bp), endoderm (AFP, F: 5' - gaa atg act cca gta aac cct ggt g - 3, R: 5' - aga ctc gtt ttg tct tct ctt ccc c - 3', 400 bp; SOX17, F: 5' - cgc acg gaa ttt gaa cag ta - 3', R: 5' - aaa aca cac cca gga caa ca - 3', 510 bp), and control (GAPDH, F: 5' - aga agg ctg ggg ctc att tg - 3', R: 5' - agg ggc cat cca cag tct tc - 3', 258 bp).

\section{RESULTS}

\section{Identification of tetraploid SCNT-hESC}

After the SCNT procedure, reconstructed egg were cultured, formed early blastocysts at Day 5, and further developed to hatching blastocysts at Day 6. The hatched blastocyst made an initial outgrowth, resembling an ESC colony, within 3 days and eventually resulted in a stable hESC line (Fig. 1). Different from several diploid SCNTderived hESCs (SCNT-hESCs), two tetraploid SCNThESCs (lines CHA-NT1: 91, XXXY,-6, t[2:6]; CHA-NT3: $92, \mathrm{XXXY},-12,+20)$ were generated and confirmed by karyotyping (Fig. 2). As the spindle apparatus of MII oocytes was clearly evident in the Polyscope image of enucleation during SCNT, it was suggested that tetraploidy may be caused by reintroduction of the remaining 1 st polar body during the SCNT procedure (Fig. 1B). In fact, nuclear DNA genotyping, using the eight Short Tandem Repeat (STR) markers, confirmed that the genotypes of both the CHANT1 and CHA-NT3 tetraploid SCNT-hESCs lines included their respective nuclear donor (DFB-1) and oocyte donor genotypes (Table 1).

\section{Characterization of tetraploid SCNT-hESc}

The growth of tetraploid SCNT-hESCs was similar to that of normal diploid SCNT-hESCs (CHA-NT2 and CHANT4) (CHA-NT2 and CHA-NT4) (Chung et al., 2014). Also, their colonies and tetraploidy were well maintained after at least 30 manual passages, although a tendency for slow growth during culture was shown. However, a small number of aneuploid cells was sometimes observed during passaging (data not shown).

To characterize the pluripotency of tetraploid SCNThESCs, we first analyzed expression of hESC-specific markers. CHA-NT1 and CHA-NT3 hESCs showed the expression of pluripotency genes, OCT-4, SSEA-4, TRA1-60, and TRA-1-81, and the RT-PCR results for OCT-4, $N A N O G$, and SOX2 confirmed expression (Fig. 3). 
A

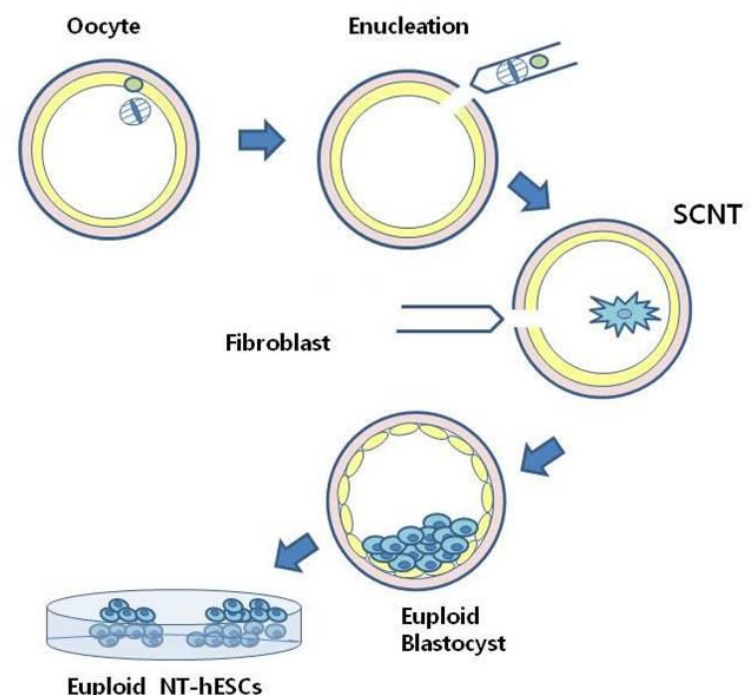

B

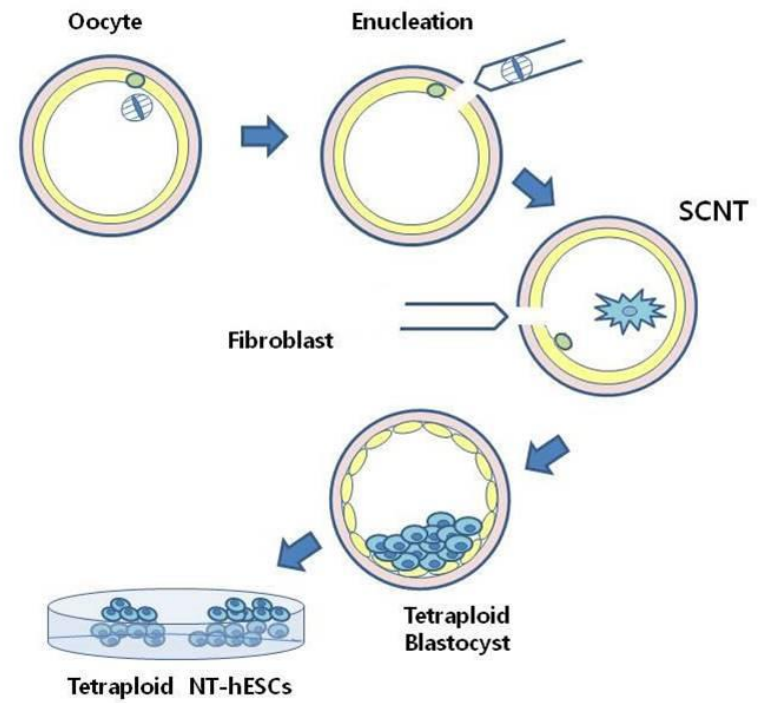

Fig. 1. Procedure for somatic cell nuclear transfer (SCNT) and derivation of embryonic stem cells (hESCs). (A) During the enucleation process, the 1st polar body was also removed. (B) During the enucleation process, the 1st polar body remained and resulted in the formation of tetraploid SCNT-hESCs.

A

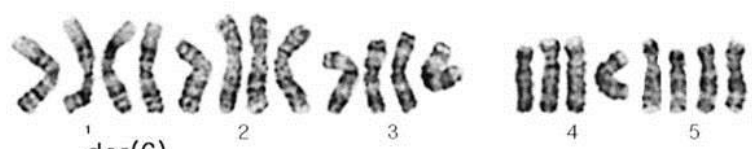
der(6)
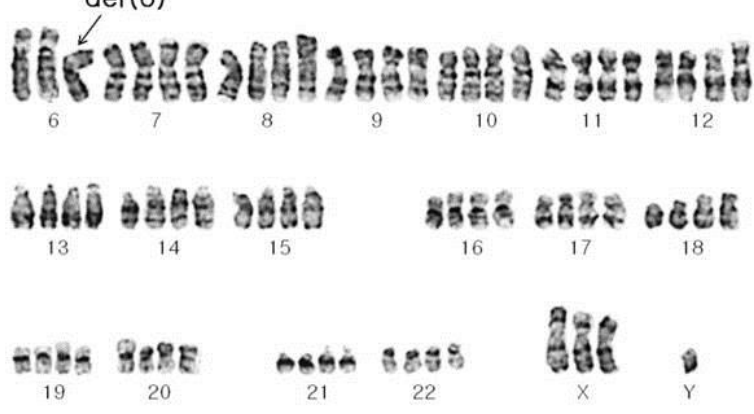

CHA-hES NT1 (Karyotyping : 91,XXXY,-6,der(6)
B
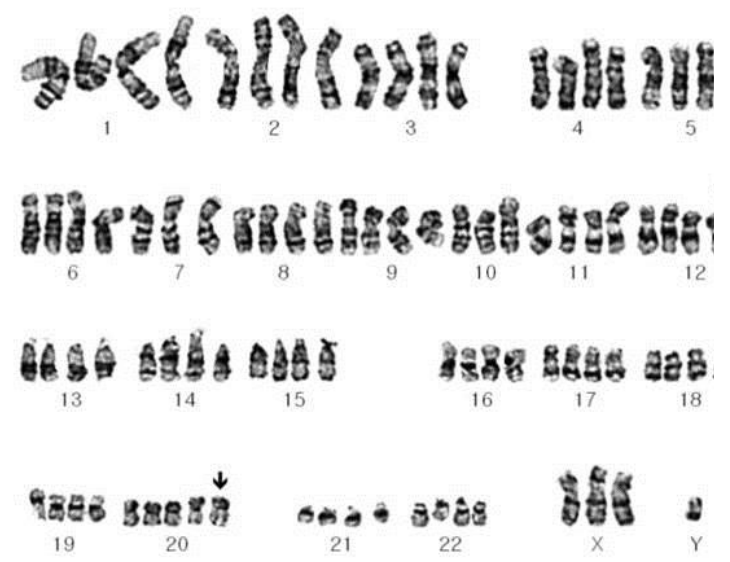

CHA-hES NT3 (Karyotyping : 92,XXXY,-12,+20)

Fig. 2. Cytogenetic G-banding analysis showed that CHA-NT1 (A) and CHA-NT3 (B) had tetraploid karyotypes.

The differentiation capacity of the tetraploid SCNThESCs lines was confirmed by EB formation in vitro and teratoma formation in vivo. As shown in Fig. 4, EBs from CHA-NT1 and CHA-NT3 hESCs showed the expression of AFP (a marker for the endoderm), aSMA (a marker for the mesoderm), and Tuji (a marker for the ectoderm). Also, the teratoma formation assay demonstrated that the tissues of the three germ layers (secretory epithelium as the endoderm, muscle fibers and cartilage as the mesoderm, and neural rosette as the ectoderm) grew out from the 
Tetraploid SCNT-ESC Lines

Table 1. Nuclear DNA genotyping using 8 STR markers (6 on autosomal loci and 2 on X/Y locus)

\begin{tabular}{|c|c|c|c|c|c|c|c|c|c|c|}
\hline \multirow{2}{*}{$\begin{array}{c}\text { STR } \\
\text { D8S1179 }\end{array}$} & \multicolumn{2}{|c|}{ DFB-1 } & \multicolumn{4}{|c|}{ CHA-NT1 } & \multicolumn{4}{|c|}{ CHA- NT3 } \\
\hline & 14 & 14 & 13 & 14 & & & 10 & 12 & 13 & \\
\hline D21S11 & 28 & 29 & 28 & 29 & 30 & 31.2 & 30 & 31 & & \\
\hline D3S1358 & 14 & 17 & 14 & 15 & 16 & 17 & 14 & 15 & & \\
\hline THO1 & 6 & 9 & 6 & 9 & & & 6 & 7 & 8 & 9.3 \\
\hline D19S433 & 15.2 & 16.2 & 13 & 15.2 & 16.2 & & 14 & 15 & 18.2 & \\
\hline vWA & 16 & 17 & 15 & 16 & 17 & & 15 & 16 & 18 & \\
\hline AMEL & $\mathrm{X}$ & Y & $\mathrm{X}$ & Y & & & $X$ & $\mathrm{Y}$ & & \\
\hline FGA & 19 & 23 & 19 & 23 & 24 & & 20 & 21 & & \\
\hline
\end{tabular}

A
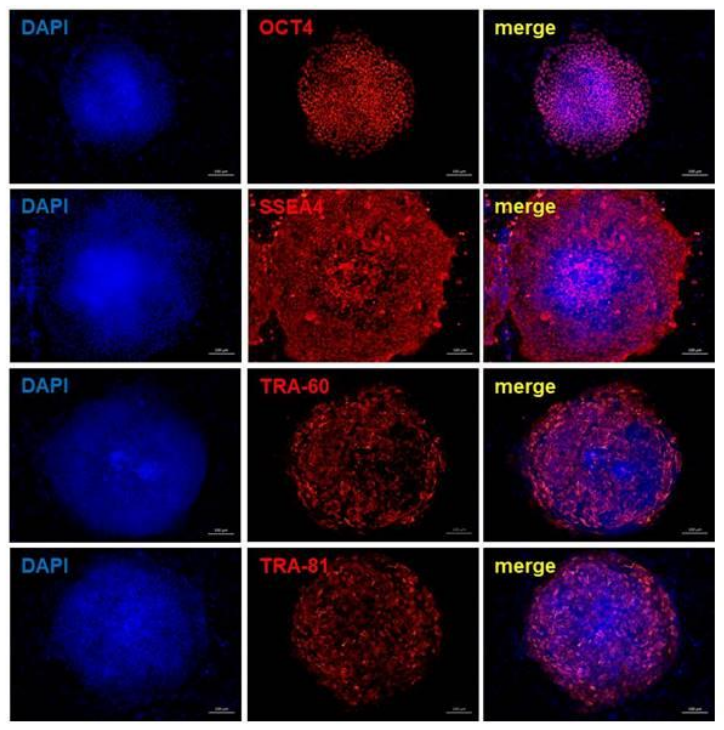

merge

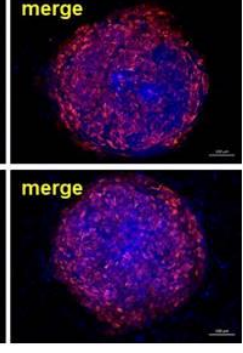

M.W. GAPDH

SOX2
536bp)

NANOG

(153bp)

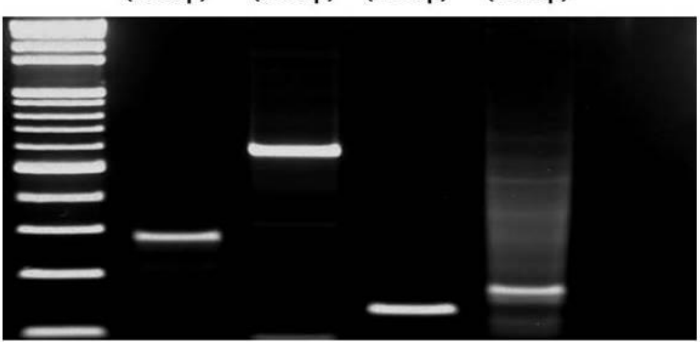

B
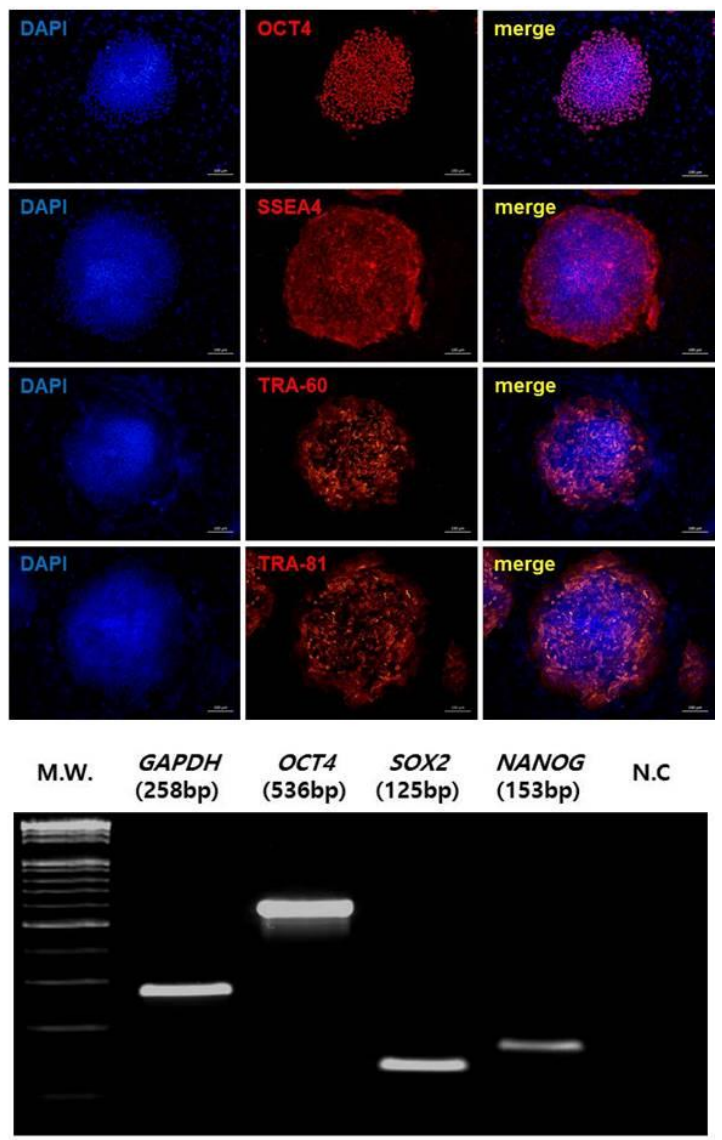

Fig. 3. Expression of pluripotency markers in CHA-NT1 (A) and CHA-NT3 hESCs (B). Immunocytochemistry for standard stem cell markers (Oct3/4, SSEA-4, TRA-1-60, and TRA-1-81) was used to show markers of undifferentiated human pluripotent stem cells (upper panels). Scale Bar $=100 \mu \mathrm{m}$. Analysis of mRNA expression in colonies of CHA-NT1 and CHA-NT3 hESCs by RT-PCR. Expression of pluripotent makers (Oct4, Sox2, and Nanog) and an internal control gene (GAPDH). N.C.: negative control (lower panels). 
A
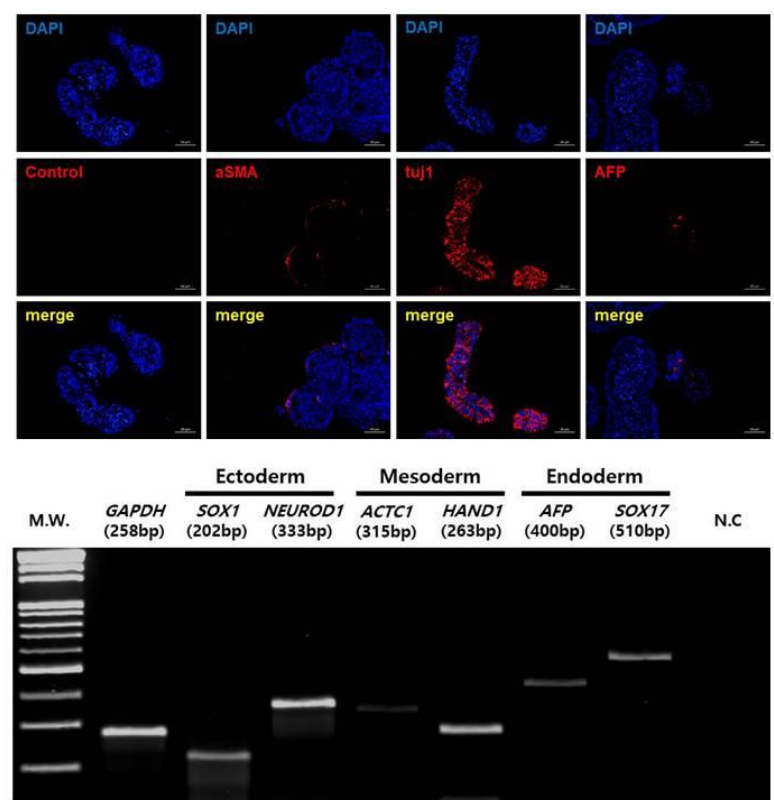

B
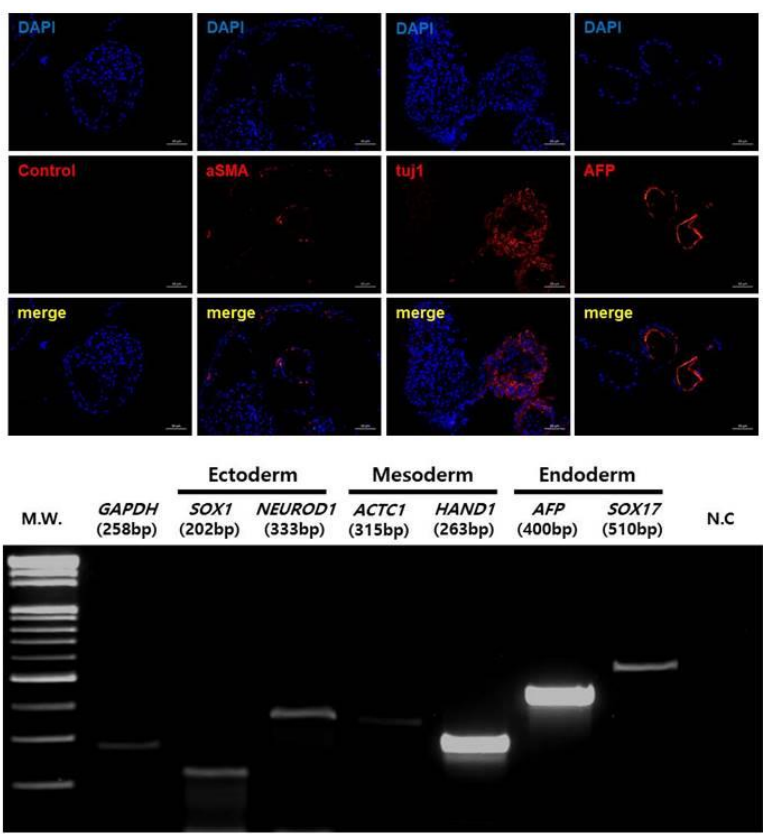

Fig. 4. Expression of three germ layer markers in embryoid bodies (EBs) of CHA-NT1 (A) and CHA-NT3 hESCs (B). Immunocytochemistry for all three germ layer markers (AFP for the endoderm, aSMA for the mesoderm, and tuj 1 for the ectoderm) was used to show markers of differentiated cells from human pluripotent stem cells (upper panels). The primary antibodies were omitted for the negative control. Scale Bar $=50 \mu \mathrm{m}$. Analysis of mRNA expression in EBs of CHA-NT1 and CHA-NT3 by RT-PCR (lower panels). Expression of differentiation markers (SOX1 and NeuroD1 for the ectoderm, ACTC1 and HAND1 for the mesoderm, AFP and SOX17 for the endoderm, GAPDH for an internal control, and N.C: negative control). RT-PCR results of EBs from CHA-NT1 and CHA-NT3.

CHA-NT1 and CHA-NT3 hESCs (Fig. 5).

\section{DISCUSSION}

In the present study, we have analyzed the characteristics and differentiating potentials of tetraploid SCNThESC lines established with traditional SCNT protocols (Chung et al., 2014). Genetic evidence, such as karyotyping and analysis of STR markers, verified the tetraploidy of CHA-NT1 and CHA-NT3, even though CHANT1 has one triple (- chromo. 6) and CHA-NT3 has two pentaploid (+ chromo. 12, 20) chromosomes. In fact, the genotypes suggested that CHA-NT1 and CHA-NT3 tetraploid SCNT-hESCs lines may have both genotypes of their respective nuclear and oocyte donors. Therefore, these re- sults may suggest that both tetraploid SCNT-hESC lines were generated by reintroduction of the remaining 1 st polar body during the SCNT procedure.

Studies on the characterization have shown that our tetraploid SCNT-hESCs lines expressed the pluripotency markers OCT-4, SSEA-4, TRA-1-60, and TRA-1-81. In addition, immunostaining of AFP, aSMA, and tuj 1 in EBs derived from tetraploid SCNT-hESC lines confirmed their potential to differentiate into all three germ layers. Also, the three germ layers were clearly visible in the CHA-NT1 and CHA-NT3 teratomas: secretory epithelium (endoderm), muscle fibers and cartilage (mesoderm), and neural rosette (ectoderm). Also, we have found that tetraploid SCNT-hESC lines maintained their ploidy well and retained stemness after passaging. Therefore, these charac- 


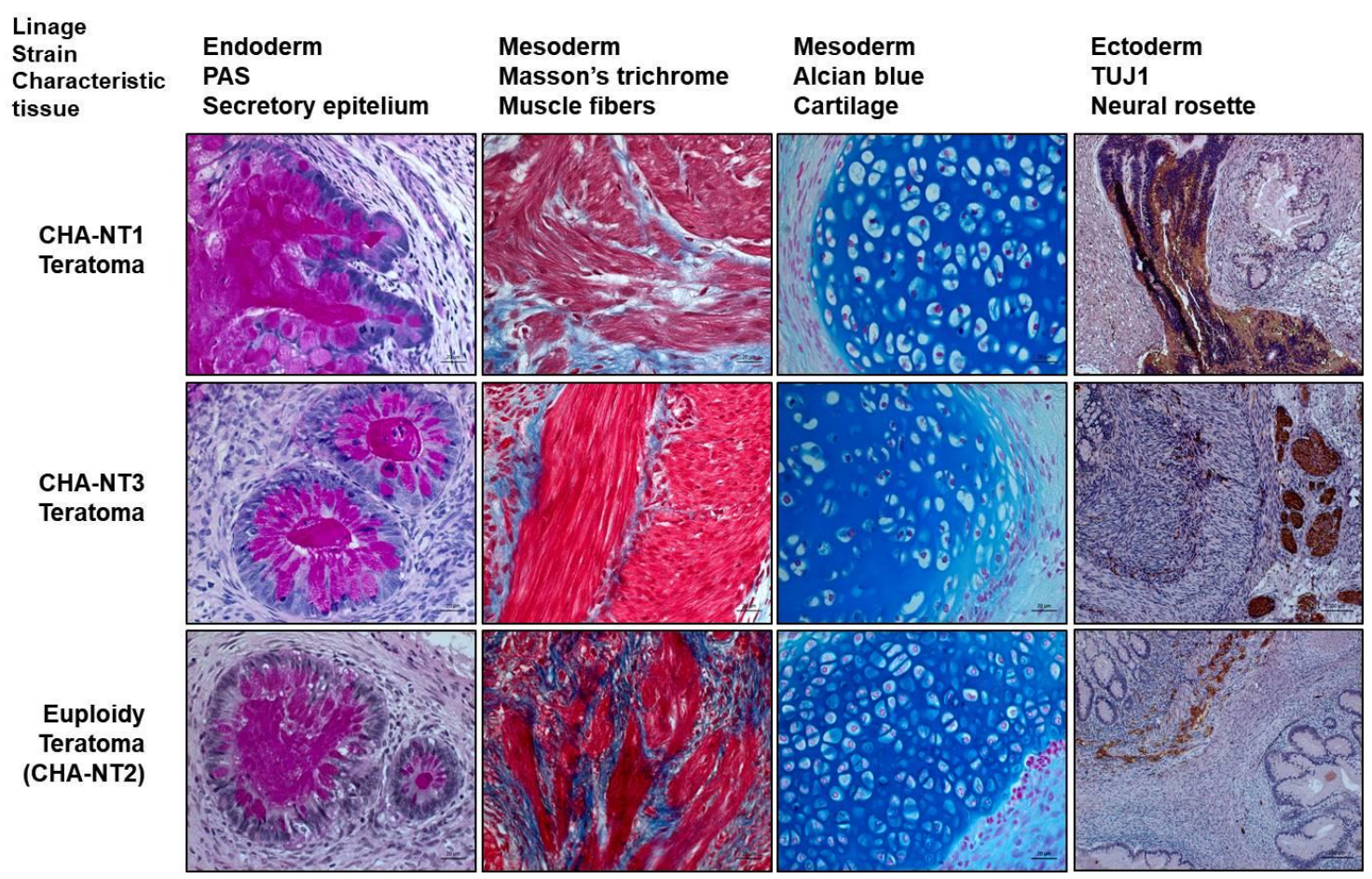

Fig. 5. Teratoma assay confirms the differentiation capacity of CHA-NT1 and CHA-NT3 hESCs: Derivatives of all three germ layers were clearly visible in teratomas, observed by the secretory epithelium for the endoderm, muscle fiber and cartilage for the mesoderm, and neural rosette for the ectoderm. The teratoma pattern of tetraploid SCNThESCs (CHA-NT1 and CHA-NT3) was similar to that of euploid SCNT-hESCs (CHA-NT2). Scale bars indicate $200 \mu \mathrm{m}$.

teristics support the idea that our tetraploid SCNT-hESCs lines could be useful for the study of tetraploid cells and tissues, because they are very similar to those of euploid SCNT-hESCs (CHA-NT2 and CHA-NT4) (Chung et al., 2014). In fact, in nature, polyploidy is very common among plants, fish, and amphibians, while polyploidy in mammals is a very rare case (Imai et al., 2015). Besides, there are several tissues with tetraploidy in mammals, including human beings. Taken together, this study identified that tetraploidy does not affect proliferation and the differentiating potential in human SCNT-hESCs. So, tetraploid SCNT-hESC lines may have the effect and enable the investigation of the characteristics of polyploid cells in humans. Also, tetraploid SCNT-hESC lines could be a useful model for polyploidy examination.

Meanwhile, in the present study, our tetraploid hESCs have shown a tendency for slow growth in stem cell cul- ture. It has been considered that tetraploidy may interfere with cell division. In fact, it has been reported that tetraploid cells have some problems in the developmental process and growth retardation, due to DNA damage and cellular metabolic stress (Storchova \& Pellman, 2004; Ganem et al., 2007). Also, tetraploid cells are highly unstable and prone to transformation. Therefore, tetraploid SCNThESC would also be a good research tool for developmental interference in pathogenic cells with abnormal ploidy, such as cancer cells.

\section{ACKNOWLEDGEMENTS}

This research was supported partly by grants from the Bio \& Medical Technology Development Program (2015 M3A9C6028961) and Basic Science Research (20090093821) of the National Research Foundation of Korea 
(NRF). The authors declare that there is no conflict of interest that could be perceived as prejudicing the impartiality of the research reported.

\section{REFERENCES}

Biesterfeld S, Gerres K, Fischer-Wein G, Bocking A (1994) Polyploidy in non-neoplastic tissues. J Clinic Pathol 47:38-42.

Chung Y, Klimanskaya I, Becker S, Li T, Maserati M, Lu SJ, Zdravkovic T, Ilic D, Genbacev O, Fisher S, Krtolica A, Lanza R (2008) Human embryonic stem cell lines generated without embryo destruction. Cell Stem Cell 2:113-117.

Chung YG, Eum JH, Lee JE, Shim SH, Sepilian V, Hong SW, Lee Y, Treff NR, Choi YH, Kimbrel EA, Dittman RE, Lanza R, Lee DR (2014) Human somatic cell nuclear transfer using adult cells. Cell Stem Cell 14:777780.

Danes BS (1976) The Gardner syndrome: increased tetraploidy in cultured skin fibroblast. J Med Genet 13:5256.

Duelli DM, Padilla-Nash HM, Berman D, Murphy KM, Ried T, Lazebnik Y (2007) A virus causes cancer by inducing massive chromosomal instability through cell fusion. Curr Biol 17:431-437.

Edgar BA, Orr-Weaver TL (2001) Endoreplication cell cycles: more for less. Cell 105:297-306.

Ganem NJ, Storchova Z, Pellman D (2007) Tetraploidy, aneuploidy and cancer. Curr Opin Genet Dev 17:157-162.

Guc-Scekic M, Milasin J, Stevanovic M, Stojanov LJ, Djordjevic M (2002) Tetraploidy in a 26-month-old girl (cytogenetic and molecular studies). Clin Genet 61: 62-65.

Hau PM, Siu WY, Wong N, Lai PB, Poon RY (2006) Polyploidization increases the sensitivity to DNA-damaging agents in mammalian cells. FEBS Lett 580:47274736.
Imai H, Kano K, Fujii W, Takasawa K, Wakitani S, Hiyama M, Nishino K, Kusakabe KT, Kiso Y (2015) Tetraploid embryonic stem cells maintain pluripotency and differentiation potency into three germ layers. PloS One 10:e0130585.

Kawaguchi J, Kano K, Naito K (2009) Expression profiling of tetraploid mouse embryos in the developmental stages using a cDNA microarray analysis. J Reprod Dev 55:670-675.

Koizumi N, Fukuta K (1995) Preimplantation development of tetraploid mouse embryo produced by cytochalasin B. Exp Anim 44:105-109.

Lafer CZ, Neu RL (1988) A liveborn infant with tetraploidy. Am J Med Genet 31: 375-378.

Lv L, Zhang T, Yi Q, Huang Y, Wang Z, Hou H, Zhang H, Zheng W, Hao Q, Guo Z, Cooke HJ, Shi Q (2012) Tetraploid cells from cytokinesis failure induce aneuploidy and spontaneous transformation of mouse ovarian surface epithelial cells. Cell Cycle 11:2864-2875.

Mable BK, Otto SP (2001) Masking and purging mutations following EMS treatment in haploid, diploid and tetraploid yeast (Saccharomyces cerevisiae). Genet Res 77: 9-26.

Mandrioli D, Belpoggi F, Silbergeld EK, Perry MJ (2016) Aneuploidy: A common and early evidence-based biomarker for carcinogens and reproductive toxicants. Environ Health 15:97.

Mazumdar M, Lee JH, Sengupta K, Ried T, Rane S, Misteli T (2006) Tumor formation via loss of a molecular motor protein. Curr Biol 16:1559-1564.

Storchova Z, Breneman A, Cande J, Dunn J, Burbank K, O'Toole E, Pellman D (2006) Genome-wide genetic analysis of polyploidy in yeast. Nature 443:541-547.

Storchova Z, Kuffer C (2008) The consequences of tetraploidy and aneuploidy. J Cell Sci 121:3859-3866.

Storchova Z, Pellman D (2004) From polyploidy to aneuploidy, genome instability and cancer. Nat Rev Mol Cell Biol 5:45-54. 